\title{
An expedited genes-to-drug approach using cryo-EM enabled structure based drug design
}

Kenneth Borrelli ${ }^{1}$, Leah Frye ${ }^{2}$ and Mazdak Radjaina ${ }^{3}$

${ }^{1}$ Schrodinger, New York, New York, United States, ${ }^{2}$ Schrodinger, United States, ${ }^{3}$ ThermoFisher, United States

Improving Protein-Ligand Modeling into Cryo-EM Data and the use of those Models in Drug Discovery Efforts Producing an accurate atomic model of protein-ligand interactions from the data generated by cryoelectron microscopy is often a challenging problem due to a combination of the noise in the experiment and the dynamic nature of protein-ligand binding. In order to address this problem we have developed ways to combine established computational modeling techniques with EM map potentials to create more accurate and more validated structural models of protein ligand binding.Here we report on the incorporation of the OPLS3e force field with the VSGB2.1 implicit solvation model into the popular Phenix package for real and reciprocal space model refinement. Our results show significantly improved structure quality at lower resolution for Xray refinement with reduced ligand strain, while showing only a slight increase in Rfree. For real space refinement of cryo-EM based structures, we find comparable quality structures, goodness-of-fit and reduced ligand strain. We will also present modifications to our Glide ligand docking software to allow it to place ligands into unmodeled density which can then be refined by Phenix. The pipeline is validated with a combination of retrospective results and application of the protocol to drug discovery efforts. Once such effort we will present is a blinded gene-to-drug pipeline in the context of a strategic collaboration between Schrodinger Inc and ThermoFisher Scientific. GeneArt ${ }^{\circledR}$ Gene-to-Proteins was used to create highly pure native protein with a concentration of $5 \mathrm{mg} / \mathrm{ml}$. The Thermo Scientific iSPA Workflow was used to solve

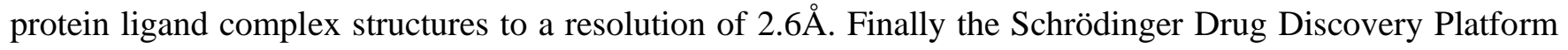
used these structures to to create a validated structure-based model of the binding affinity of congeneric ligands capable of correctly separating strong binders $(<100 \mathrm{nM})$, intermediate binders $(100 \mathrm{nM}-1 \mathrm{uM})$ and weak binders $(<1 \mathrm{uM})$ with $73 \%$ accuracy from a set of 62 previously patented ligands. The entire workflow from a gene to a validated affinity model that was guiding synthesis decisions was completed in a few months. 


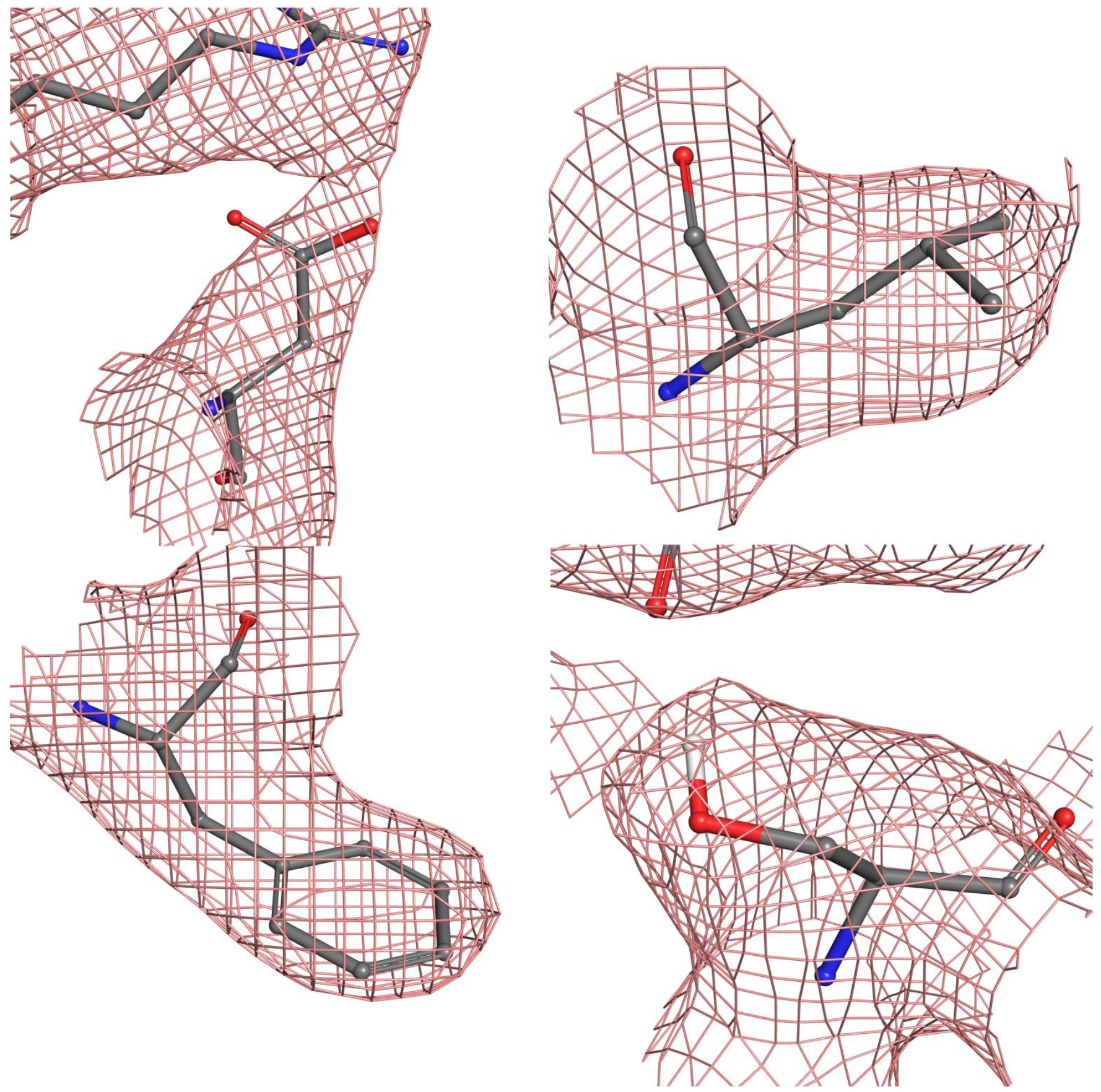

Figure 1. Detail of 4 representative residues showing the coulombic potential reconstructed from the EM data collection and the fitted model. As can be seen, the data was sufficient to ascertain the position of most sidechains. 


\section{Predicted Affinity}

\begin{tabular}{|c|c|c|c|}
\hline & $<100 \mathrm{nM}$ & $100 \mathrm{nM}-1 \mathrm{uM}$ & $>1 \mathrm{uM}$ \\
\hline$<100 \mathrm{nM}$ & 6 & 6 & 0 \\
\hline 100nM - 1um & 4 & 20 & 6 \\
\hline$>1 \mathrm{uM}$ & 0 & 7 & 35 \\
\hline \multicolumn{4}{|c|}{$\begin{array}{l}\text { Strict Accuracy: } 73 \% \text { ( Green Boxes ) } \\
\text { Off By One Accuracy: } 100 \% \text { ( Green + Yellow Boxes ) }\end{array}$} \\
\hline
\end{tabular}

Figure 2. FEP+ predicted vs measured affinity for a series of 62 previously patented congeneric ligands enabled by a cryo-EM structure of the target protein solved for this effort in complex with a member of the congeneric set.

\section{References}

GemSpot: A Pipeline for Robust Modeling of Ligands into CryoEM Maps

Michael J. Robertson, Gydo C. P. van Zundert, Kenneth Borrelli, Georgios Skiniotis bioRxiv 750778; doi: https://doi.org/10.1101/750778

Li, Qi; Pellegrino, Jenna; Lee, D. John; Tran, Arthur; Wang, Ruoxi; Park, Jesslyn; et al. (2019): Synthesis and Mechanism of Action of Group a Streptogramin Antibiotics That Overcome Resistance. ChemRxiv. Preprint. https://doi.org/10.26434/chemrxiv.8346107.v2

Wei J, Leit S, Kuai J, Therrien E, Rafi S, Harwood HJ Jr, DeLaBarre B, Tong L. An allosteric mechanism for potent inhibition of human ATP-citrate lyase. Nature. 2019 Apr;568(7753):566-570. 